\title{
Non-invasive monitoring of arthritis treatment response via targeting of tyrosine- phosphorylated annexin A2 in chondrocytes
}

Shaw-Wei D. Tsen ${ }^{1}$, Luke E. Springer ${ }^{2}$, Krishna Sharmah Gautam', Rui Tang ${ }^{1}$, Kexian Liang ${ }^{1}$, Gail Sudlow', Amir Kucharski ${ }^{1}$, Christine T. N. Pham ${ }^{2^{*}}$ and Samuel Achilefu ${ }^{1,3,4^{*}}$ (1)

\begin{abstract}
Background: The development and optimization of therapies for rheumatoid arthritis (RA) is currently hindered by a lack of methods for early non-invasive monitoring of treatment response. Annexin $A 2$, an inflammation-associated protein whose presence and phosphorylation levels are upregulated in RA, represents a potential molecular target for tracking RA treatment response.
\end{abstract}

Methods: LS301, a near-infrared dye-peptide conjugate that selectively targets tyrosine 23-phosphorylated annexin A2 (pANXA2), was evaluated for its utility in monitoring disease progression, remission, and early response to drug treatment in mouse models of RA by fluorescence imaging. The intraarticular distribution and localization of LS301 relative to PANXA2 was determined by histological and immunohistochemical methods.

Results: In mouse models of spontaneous and serum transfer-induced inflammatory arthritis, intravenously administered LS301 showed selective accumulation in regions of joint pathology including paws, ankles, and knees with positive correlation between fluorescent signal and disease severity by clinical scoring. Whole-body near-infrared imaging with LS301 allowed tracking of spontaneous disease remission and the therapeutic response after dexamethasone treatment. Histological analysis showed preferential accumulation of LS301 within the chondrocytes and articular cartilage in arthritic mice, and colocalization was observed between LS301 and pANXA2 in the joint tissue.

Conclusions: We demonstrate that fluorescence imaging with LS301 can be used to monitor the progression, remission, and early response to drug treatment in mouse models of RA. Given the ease of detecting LS301 with portable optical imaging devices, the agent may become a useful early treatment response reporter for arthritis diagnosis and drug evaluation.

Keywords: Near-infrared fluorescent imaging, Treatment response monitoring, Inflammatory arthritis, Rheumatoid arthritis

*Correspondence: cpham@wustl.edu; achilefu@wustl.edu

${ }^{1}$ Departments of Radiology, Washington University School of Medicine, St Louis, MO 63110, USA

2 Division of Rheumatology, Washington University School of Medicine, St Louis, MO 63110, USA

Full list of author information is available at the end of the article

\section{Background}

Rheumatoid arthritis (RA) is among the most common debilitating joint conditions in the United States, affecting up to $1 \%$ of the population [1-4]. In recent decades, therapeutic advances in disease-modifying antirheumatic drugs (DMARDs) have enabled inhibition of disease progression and made clinical remission an achievable goal [5-8]. However, a challenge remains 
in optimizing treatment regimens to reach such a state in the shortest duration of time, to minimize damage to the joints caused by elevated disease activity.

There is currently a lack of methods for monitoring early treatment response in RA patients, which has hampered accurate assessment of disease activity and posed a significant barrier to treatment adjustment [9]. In the context of RA, successful clinical management relies on proper selection of a therapy to which the patient will show response [10]. Unfortunately, the response to any particular drug is variable among patients, and there are no well-established methods to guide therapeutic choices [11]. The current clinical paradigm involves a series of trials and errors, where treatment response is monitored over a course of months relying on clinical scoring methods and imaging modalities such as X-rays and ultrasonography $[10,12,13]$, which are insensitive to very early (i.e., $<1$ month) changes in disease activity. Positron emission tomography (PET), an experimental approach for arthritis imaging, provides greater sensitivity but has the disadvantage of increased radiation exposure [14]. Together, these limitations lead to additional costs and toxicities to the patient, and potentially worsens patient outcomes, since early treatment is ideal for optimal reduction in joint damage [15]. Importantly, the speed with which novel arthritis therapies can be evaluated in clinical trials depends on feedback regarding treatment efficacy. In these regards, there is a need for a non-invasive method that enables rapid assessment of therapeutic effect for RA.

Recently, fluorescence imaging (FI) has been explored as a novel method for diagnosis and tracking of arthritis treatment response in both preclinical models and humans [16-24]. The technique involves the administration of a near-infrared fluorophore, followed by the detection of accumulated fluorescence in affected joints. Near-infrared FI has several advantages over conventional imaging modalities including its low cost and the avoidance of ionizing radiation exposure. FI is well-suited for application to RA, where peripheral small joints in the extremities are involved. Agents that have been utilized in prior studies include non-targeted dyes (e.g. indocyanine green (ICG), Cy5.5) [18, 24]; dye-labeled monoclonal antibodies and small molecule ligands that bind macrophage or endothelial cell targets such as $\mathrm{F} 4 / 80$, E-selectin, $\alpha \mathrm{v} \beta 3$ integrin, and folate receptor $\mathrm{s}$ [25-28]; and enzyme-activatable probes. Non-targeted dyes such as ICG accumulate in inflamed joints primarily due to increased vascular permeability, resulting in low contrast in comparison to targeted agents $[29,30]$. On the other hand, the existing targeted approaches which focus on activated macrophages and endothelial cells have reduced specificity as these cell types are not limited to RA.
We have developed a novel near-infrared dye-peptide conjugate termed LS301 (Fig. S1), which preferentially binds Tyr23-phosphorylated annexin A2 protein (pANXA2) with high affinity [31]. Annexin A2 is a calciumdependent phospholipid-binding protein that plays a key role in inflammation by facilitating NF- $\mathrm{kB}$ activation and plasmin-dependent macrophage migration and infiltration in association with its binding partner S100A10 on the cell surface [32,33]. Phosphorylation of annexin A2 at Tyr23 is a prerequisite for its translocation to the cell surface $[32,34,35]$, where it is amenable to targeting by exogenously administered agents such as LS301. The expression and phosphorylation levels of annexin A2 are known to be upregulated in human RA patients relative to healthy controls, and overexpression of annexin $\mathrm{A} 2$ in joints promotes RA disease progression via induction of angiogenesis and joint destruction [36, 37]. Therefore, pANXA2 represents a potential target for molecular imaging of RA. In this report, we investigated the utility of pANXA2-targeted fluorescence imaging using LS301 for monitoring early treatment response in experimental inflammatory arthritis.

\section{Methods \\ Chemicals and reagents}

All the fluorenylmethyloxycarbonyl (Fmoc) amino acids and Fmoc-Lys (Boc)-Wang Resin were purchased from AAPPTec (Louisville, KY, USA). Dichloromethane (DCM), acetic acid, acetic anhydride, thioanisole, phenol, hydroxybenzotriazole (HOBt), N,N-diisopropylethylamine (DIEA), N-trityl-1,2-ethanediamine, phenol, thioanisol, dimethylformamide (DMF), N, $\mathrm{N}^{\prime}-$ diisopropylcarbodiimide (DIC), trifluoroacetic acid (TFA), iodine, methyl tert-butyl ether (MTBE), O-(7azabenzotriazol-1-yl)-N,N, $\mathrm{N}^{\prime}, \mathrm{N}^{\prime}$-tetramethyluronium hexafluorophosphate (HATU), and dexamethasone (DEX) were purchased from Sigma-Aldrich (St Louis, MO). Hematoxylin and eosin (H\&E) stains were purchased from MilliporeSigma (St Louis, MO). Rabbit antipANXA2 (phospho-Tyr24) antibody was purchased from Signalway Antibody (College Park, MD). AlexaFluor 594-conjugated donkey anti-rabbit antibody was purchased from Thermo Fisher Scientific (Waltham, MA).

\section{Synthesis of LS301}

LS301 (cypate-cyclic ( ${ }^{\mathrm{D} C y s-G l y-A r g-A s p-S e r-P r o-C y s)-~}$ Lys-OH) was synthesized as previously reported [31]. Briefly, the linear GRD peptide, $\mathrm{H}^{-}{ }^{\mathrm{D}} \mathrm{Cys}$ (Acm)-Gly-Arg (Pbf)-Asp (tBu)-Ser (tBu)-Pro-Cys (Acm)-Lys (Boc)-OH, was prepared via a CEM Liberty Blue microwave peptide synthesizer (Matthews, NC, USA) on the Fmoc-Lys (Boc)wang resin. The resin $(0.1 \mathrm{mmol})$ was swelled in DCM for $1 \mathrm{~h}$ before use. Fmoc-amino acids $(0.5 \mathrm{mmol}, 5 \mathrm{eq})$, coupling reagent (HBTU, $0.5 \mathrm{mmol}, 5 \mathrm{eq}$ ), and DIEA (1 
mmol, 10 eq) were added to the resin and the mixture was reacted for $15 \mathrm{~min}$ under microwave irradiation $(100 \mathrm{~W}$, $90^{\circ} \mathrm{C}$ ). The resin was washed three times with DMF. Deprotection of Fmoc group was carried out by treatment of $20 \%$ piperidine/DMF for 5 min under microwave irradiation $\left(100 \mathrm{~W}, 90^{\circ} \mathrm{C}\right)$. The peptidyl resin was washed and the peptide cyclized through the disulfide bridge with iodine (1.2 eq) in DMF for $90 \mathrm{~min}$. Subsequently, cypate (3 eq) was conjugated to the cyclic peptide on solid support in the presence of DIC (5 eq) in DMF to afford the LS301 peptidyl resin. The resin was then treated with a cleavage cocktail of TFA: thioanisol: phenol: water (85:5:5:5, v/v/ $\mathrm{v} / \mathrm{v}$ ) for $90 \mathrm{~min}$ at room temperature. The cleaved peptide product was concentrated in vacuo before purification by reverse-phase HPLC (Gilson, Middleton, WI, USA). Analytical HPLC was used to determine product purity (> 95\%) and the compound identity was confirmed by electrospray ionization mass spectrometry on a Shimadzu LCMS-2020 Mass Spectrometer (Columbia, MD) with peaks observed at $1470(\mathrm{M}+1)$ and $735(\mathrm{M}+2 / 2)$.

\section{Animals}

Male 5-7-week-old C57BL/6J mice were purchased from The Jackson Laboratory (Bar Harbor, ME) and housed in designated animal facilities. Mice were fed ad libitum and inspected regularly. All animal experiments were performed in compliance with guidelines and protocols approved by the Division of Comparative Medicine at Washington University in St. Louis. The animal protocol is subjected to annual review and approval by The Animal Studies Committee of Washington University.

\section{Arthritis mouse models}

The K/BxN mice with spontaneous arthritis (F1) [38-40] were maintained in the laboratory of Dr. Christine Pham (Department of Internal Medicine, Washington University School of Medicine). To establish serum transfer arthritis (STA), male 6-8 weeks old C57BL/6J mice (The Jackson Lab, Bar Harbor, ME, USA) were injected intraperitoneally with $150-175 \mu \mathrm{L}$ of serum derived from $\mathrm{F} 1$ mice (8-9 weeks old), with day 0 denoting the day of serum transfer/disease induction. Clinical manifestation of arthritis in each paw was assessed daily on a scale of $0-3$ with $0=$ no swelling or erythema, $1=$ slight swelling or erythema, $2=$ moderate erythema and swelling in multiple digits or entire paw, and $3=$ pronounced erythema and swelling of an entire paw, with a maximum score of 12 per mouse as previously described [41]. Ankle thickness of two hind paws was measured using calipers. Animals were monitored for signs of distress during arthritis induction including their ability to move around the cage and access food/water.

\section{In vivo imaging}

Animals were shaved and excess hair removed using commercially available hair removal cream. Mice were anesthetized with isoflurane for injection and imaging procedures. LS301 stock (or LS301-methotrexate or LS301-methylpresdnisolone conjugate stock) in dimethyl sulfoxide solution was diluted in phosphate-buffered saline to a final concentration of $60 \mu \mathrm{M}$ and injected via tail vein into mice. In vivo near-infrared fluorescence using $785 \mathrm{~nm}$ excitation and 820 $\mathrm{nm}$ emission filters was assessed pre-injection, post-injection, and/or at indicated time points post-injection with a Pearl Small Animal Imaging System (LICOR Biotechnology, Lincoln, NE). Regions of interest (ROIs) for fluorescence quantitation were drawn and analyzed using the Pearl Small Animal Imaging System software.

\section{Treatment response monitoring studies}

Experiments were performed in a blinded fashion, where the technician responsible for clinical assessment of paw score and ankle measurements was blinded to treatment groups. For studies on disease remission, mice with STA were injected with $6 \mathrm{nmol}$ of intravenous LS301 on day 4 post-disease induction and imaged at $18 \mathrm{~h}$ post LS301 injection using the Pearl Small Animal Imaging System as described above. Clinical paw scores and ankle measurements were obtained daily. On day 23 postdisease induction when the clinical paw scores of mice were near the baseline, mice were imaged again with 6 nmol intravenous LS301. Regions of interest (ROI) were quantitated, encompassing mouse upper extremities (all structures including and distal to the wrist) and lower extremities (all structures including and distal to the ankle), applied universally to all images using the Pearl software. Total extremity fluorescence (quantitated from ROIs) per mouse, averaged among $n=3$ mice, was compared between groups. For studies on response to DEX treatment, mice with STA were injected with $6 \mathrm{nmol}$ of intravenous LS301 on day 3 post disease induction and imaged at 18h post LS301 injection using the Pearl Small Animal Imaging System with $\lambda=785 \mathrm{~nm}$ (excitation) $/ 820$ $\mathrm{nm}$ (emission). Mice then received intraperitoneal DEX $(10 \mathrm{mg} / \mathrm{kg} /$ dose $)$ daily over a 6 -day period. Clinical paw scores and ankle measurements were obtained daily. On day 9 (day of final DEX treatment), mice were imaged again with $6 \mathrm{nmol}$ intravenous LS301. Regions of interest (ROI) were quantitated and analyzed as described above.

\section{Histological assessment}

H\&E staining, immunohistochemical staining for pANXA2, and microscopic analysis were performed as previously described [31]. Tissues of interest were harvested and frozen at $-80^{\circ} \mathrm{C}$ in Optimal Cutting 


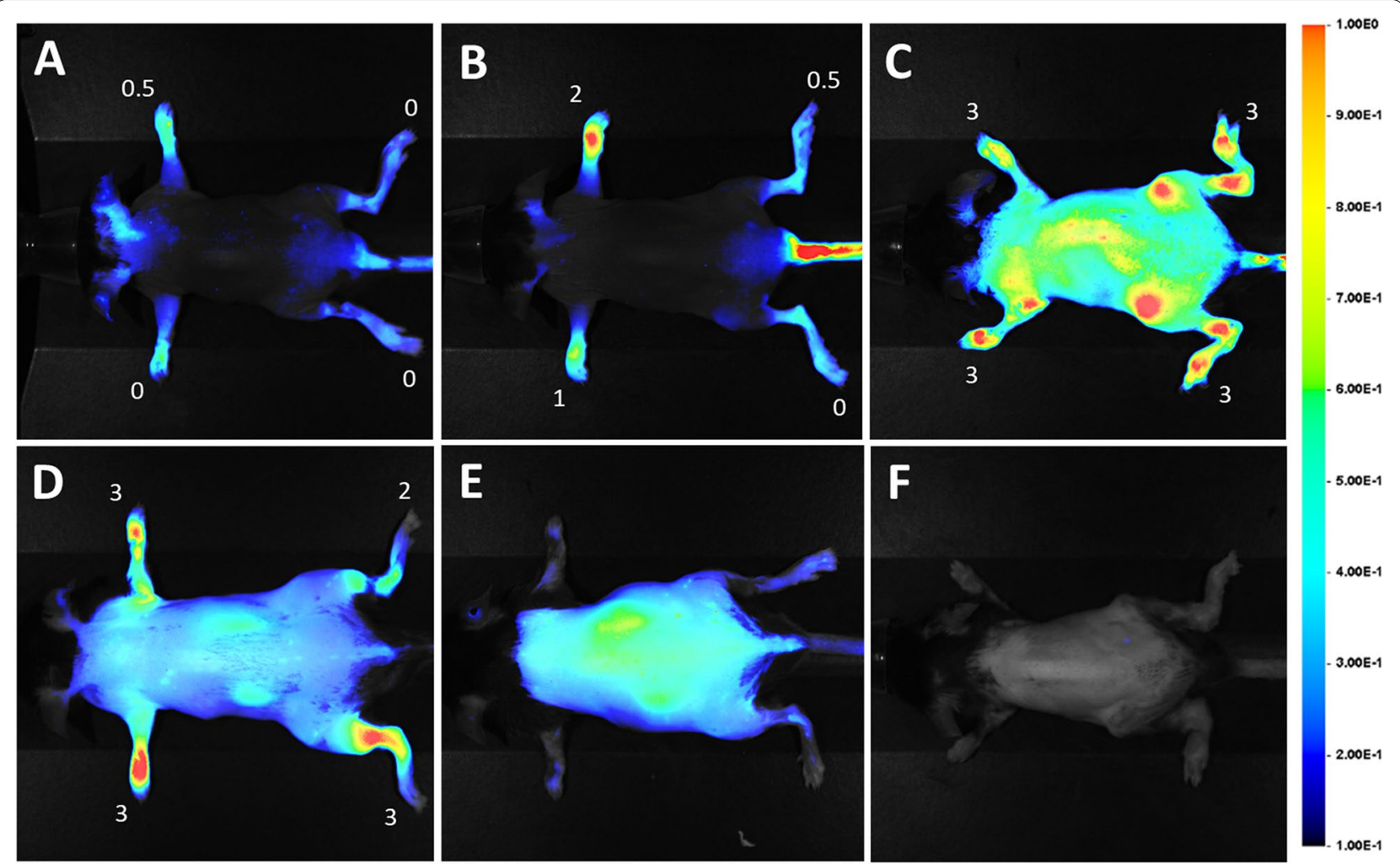

Fig. 1 In vivo imaging of K/BxN spontaneous and serum transfer arthritis using LS301. Arthritic or control (non-diseased) mice were injected intravenously with $6 \mathrm{nmol}$ LS301. Whole body near-infrared fluorescence images were taken on the Pearl animal imaging system with $\lambda=785$ $\mathrm{nm}$ excitation and $\lambda=820 \mathrm{~nm}$ emission filters. Images shown are representative of at least two independent experiments and were taken at $18 \mathrm{~h}$ post-injection with mice in dorsal orientation. A Early-stage spontaneous K/BxN arthritis (3-4 weeks old F1 mice). B Intermediate stage spontaneous K/BxN arthritis (5-7 weeks old F1 mice). C Late-stage spontaneous K/BxN arthritis (9-10 weeks old F1 mice). D C57BL/6J mice with serum transfer arthritis (day 4 post disease induction). E Normal (control) C57BL/6J mice injected with LS301. F Intermediate stage spontaneous K/BxN arthritis (5-7 weeks old) F1 mice injected with cypate dye (control) in lieu of LS301. Numbers denote individual clinical paw scores at the time of imaging

Temperature (OCT) media. Frozen sections were cut at $10 \mu \mathrm{m}$ thickness, and slides were stored at -40 ${ }^{\circ} \mathrm{C}$. Consecutive sections were subjected to H\&E and immunohistochemical analysis as follows. H\&E staining was performed by the Musculoskeletal Histology and Morphometry Core, Washington University School of Medicine. Briefly, frozen sections were fixed for 10 min in 4\% paraformaldehyde solution (Sigma, St. Louis, MO, USA) and stained with Harris hematoxylin for $90 \mathrm{~s}$ and with eosin (Sigma, St. Louis, MO) for $15 \mathrm{~s}$, and then washed with tap water for $5 \mathrm{~min}$. Some sections were stained with Safranin O and Fast Green counterstain (Musculoskeletal Histology and Morphometry Core, Washington University School of Medicine, St Louis, MO. For immunohistochemistry, slides were blocked with appropriate serum for $35 \mathrm{~min}$ or with $5 \%$ non-fat milk PBS (pH 7.4) overnight at $4{ }^{\circ} \mathrm{C}$ and incubated with primary antibody overnight at $4{ }^{\circ} \mathrm{C}$ or $1 \mathrm{~h}$ at $37^{\circ} \mathrm{C}$. For pANXA2 studies, tissue sections were incubated with 1: 250 rabbit anti-pANXA2 (phospho-Tyr24) antibody
(Signalway Antibody, College Park, MD). After washing twice with PBS, the tissue sections were incubated with 1:1000 AlexaFluor 594-conjugated donkey antirabbit antibody (Thermo Fisher Scientific, Waltham, MA) for $1 \mathrm{~h}$ at $25{ }^{\circ} \mathrm{C}$ respectively. Slides were washed again and stained with DAPI nuclear stain for $5 \mathrm{~min}$ (Thermo Fisher Scientific, Waltham, MA) for $45 \mathrm{~min}$ at $37^{\circ} \mathrm{C}$. After final washes, a coverslip with aqueous fluorescence-saving mounting media was applied prior to imaging. Slides were viewed using an Olympus B61 epifluorescence microscope (Olympus Corp., Tokyo, Japan) with filters/channels as follows: DAPI (Ex/Em $=330-385 / 420 \mathrm{~nm})$, FITC $(\mathrm{Ex} / \mathrm{Em}=460-500 / 510-$ $560 \mathrm{~nm})$, Texas Red $(\mathrm{Ex} / \mathrm{Em}=542-582 / 604-644 \mathrm{~nm})$, cypate $(\mathrm{Ex} / \mathrm{Em}=750-800 / 818-873 \mathrm{~nm})$, using exposure times 1 to $30 \mathrm{~s}$ and sensitivity settings ISO200ISO1600, with the same parameters used for control and treatment groups. ImageJ software (National Institutes of Health, Bethesda, MD, USA) was used for image processing. 

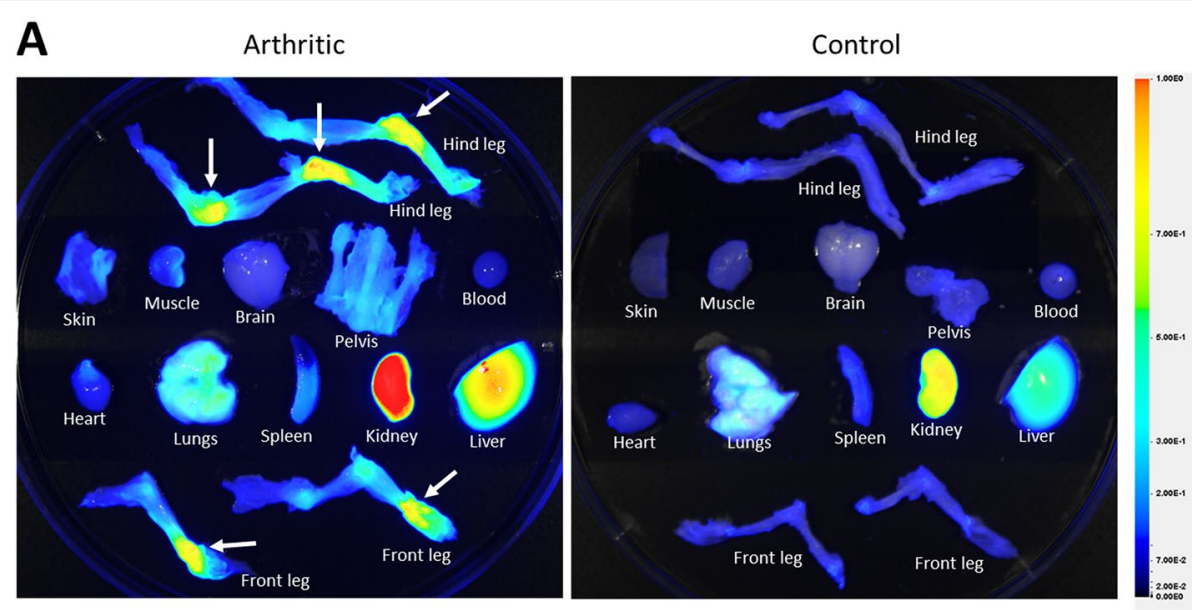

B

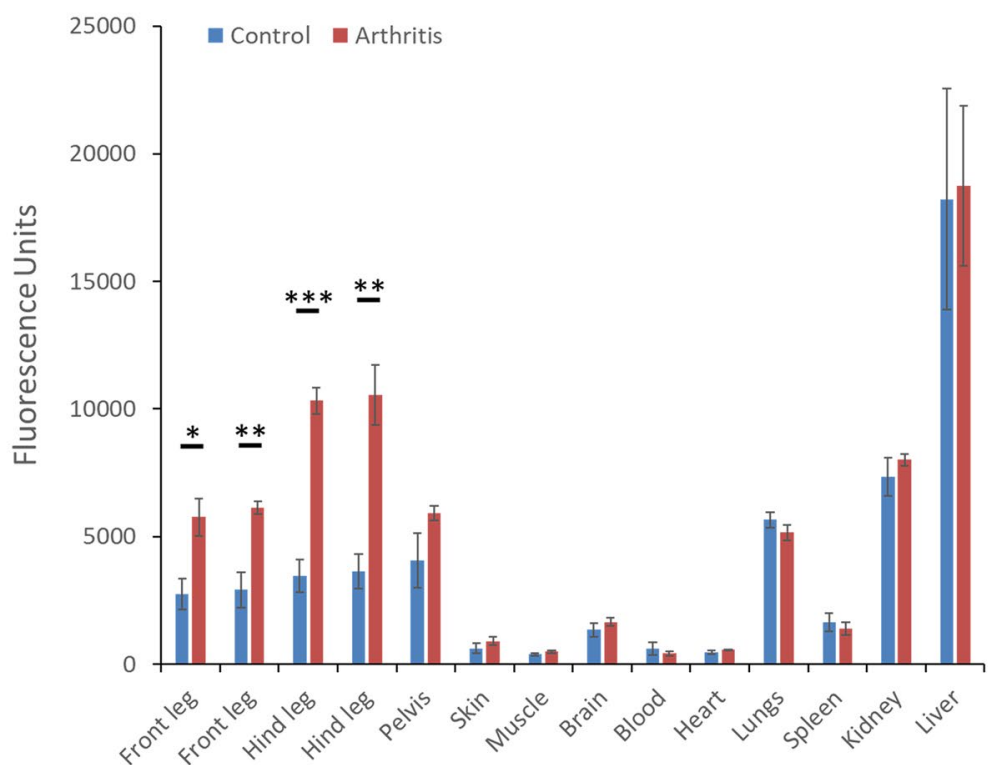

Fig. 2 Typical LS301 biodistribution in arthritic mice. A Example ex vivo organ biodistribution of LS301 in C57BL/6J mice with serum transfer arthritis (left) or in control C57BL/6J mice (right) 18h after intravenous injection, as assessed by near-infrared fluorescence imaging on the Pearl animal imaging system. C57BL/6J mice with serum arthritis (days 5-6 post serum transfer) (left) or control C57BL/6J mice (right) were injected intravenously with $6 \mathrm{nmol}$ LS301. Organs were harvested at $18 \mathrm{~h}$ post injection. Near-infrared fluorescence images were taken on the Pearl animal imaging system with $\lambda=820 \mathrm{~nm}$. Arrows denote detected areas of joint inflammation. B Quantification of fluorescence from individual organs from (A) $(n=4$ mice per group). ROls were drawn around each organ/limb of interest and quantitated using the Pearl animal imaging system software

\section{Immunoblotting}

Tissues were homogenized using an ultrasonic processor in RIPA buffer (20mM Tris-HCl, pH 7.5, 150mM NaCl, 1mM $\mathrm{Na}_{2}$ EDTA, 1mM EGTA, 1\% NP-40, 1\% sodium deoxycholate, $2.5 \mathrm{mM}$ sodium pyrophosphate, $1 \mathrm{mM}$ b-glycerophosphate, $1 \mathrm{mM} \mathrm{Na} \mathrm{VO}_{4}, 1 \mu \mathrm{g} / \mathrm{ml}$ leupeptin, $1 \mathrm{mM}$ PMSF). The tissue lysates were clarified by centrifugation. The protein was denatured in SDS gel-loading buffer $(100 \mathrm{mM}$ Tris$\mathrm{HCl}, 200 \mathrm{mM}$ DTT, 4\% SDS, $0.2 \%$ bromophenol blue, and
$20 \%$ glycerol) at $95{ }^{\circ} \mathrm{C}$ for $10 \mathrm{~min}$ and then separated on $12 \%$ SDS-polyacrylamide gels $(50 \mu$ g of the tissue protein per sample). After electrophoresis, proteins were transferred to PVDF membrane using an EC140 Mini Blot Module (Thermo EC, Holbrook, NY) apparatus. The membrane was blocked for $1 \mathrm{~h}$ at room temperature in PBS containing $5 \%$ nonfat dry milk (w/v), 0.1\% (v/v) Tween-20 (PBS-T), followed by incubation with Annexin A2 rabbit mAb (1:2000; Cat. 8235, Cell Signaling Tech.) or p-Annexin A2 mouse 


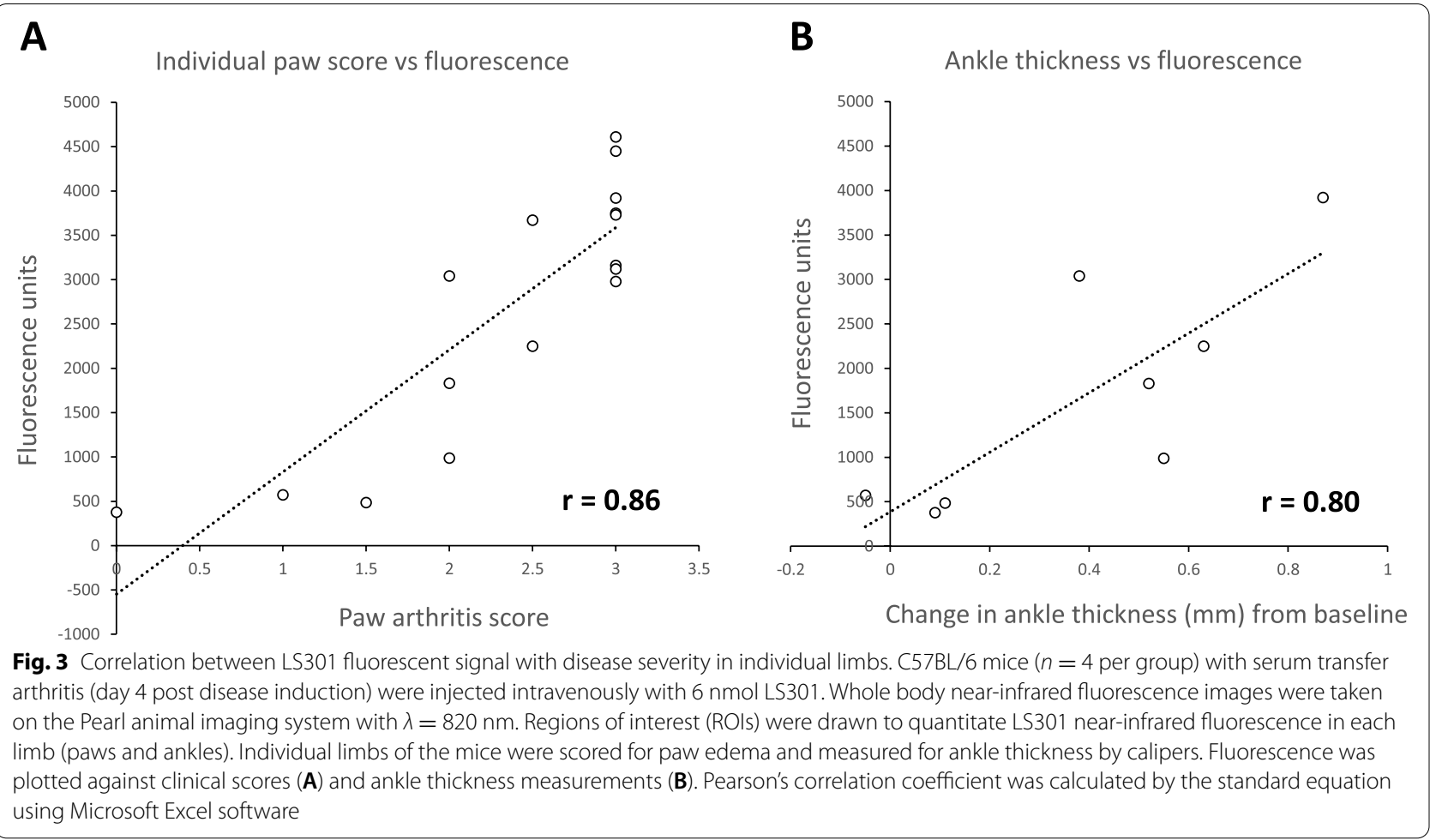

mAb (1:500; sc-135753, Lot\# J2920; Santa Cruz) in PBS-T containing $3 \%$ nonfat dry milk (w/v) at $4^{\circ} \mathrm{C}$ overnight. After washing three times for $10 \mathrm{~min}$ each in PBS-T, the membrane was incubated for $1 \mathrm{~h}$ with diluted polyclonal goat anti-rabbit IgG or polyclonal goat anti-mouse IgG conjugated to horseradish peroxidase in PBS-T containing 3\% nonfat dry milk $(\mathrm{w} / \mathrm{v})$. The membrane was then washed three times for 10 min each in PBS-T and developed using the chemiluminescence ECL kit (Pierce) according to the manufacturer's instructions.

\section{Statistics}

Differences between sample means were analyzed by two-tailed unpaired $t$-test (Microsoft Excel) with $p<0.05$ as the threshold for statistical significance. Correlations between fluorescence measurements, clinical paw scores, and change in ankle thickness were analyzed using Pearson correlation (Microsoft Excel). For semiquantitative analysis of fluorescence, 3 mice per group allows $80 \%$ power to detect an effect size of 1.67 by 2 -sided 2 -sample $t$-test at alpha $=5 \%[42]$.

\section{Results}

\section{LS301 localizes to sites of joint inflammation in models} of RA

In the $\mathrm{K} / \mathrm{BxN}$ (F1) mice with spontaneous arthritis, inflammation occurs progressively in the paws, ankle, and knee joints leading to measurable local erythema and swelling (see Methods) [41]. F1 mice with severe arthritis (9-10 weeks old) were injected intravenously with LS301 and imaged 18h post-injection for near-infrared fluorescence using the Pearl Small Animal Imaging System. A time course assessment revealed the time point of optimal contrast at $\sim 18 \mathrm{~h}$ post injection (Fig. S2), when LS301 was clearly seen to accumulate in regions of expected joint pathology in the extremities including paws and ankles. Using this time point, fluorescence imaging using LS301 in F1 mice with early (3-4 weeks old), intermediate (5-7 weeks old), or late-stage arthritis (9-10 weeks old) showed a positive correlation between fluorescence signal and disease severity as assessed by clinical paw scoring (Fig. $1 \mathrm{~A}-\mathrm{C}$ ). Similar results were obtained in mice with $\mathrm{K} / \mathrm{BxN}$ serum-transfer arthritis (STA) (Fig. 1D). Ex vivo biodistribution studies of LS301 in mice with induced arthritis confirmed selective accumulation of the agent in ankle and paw regions, with total fluorescence in individual limbs comparable to that of organs of excretion (liver, kidney) (Fig. 2). In contrast, healthy (non-diseased) control mice showed minimal LS301 accumulation in extremities (Figs. 1E and 2). To exclude increased blood flow/impeded circulation as the primary factor leading to compound accumulation in the diseased sites, we administered cypate dye alone (the dye component of LS301) to F1 arthritic mice (3-4 weeks old) and found no evidence of accumulation in extremities at $18 \mathrm{~h}$ (Fig. 1F). 


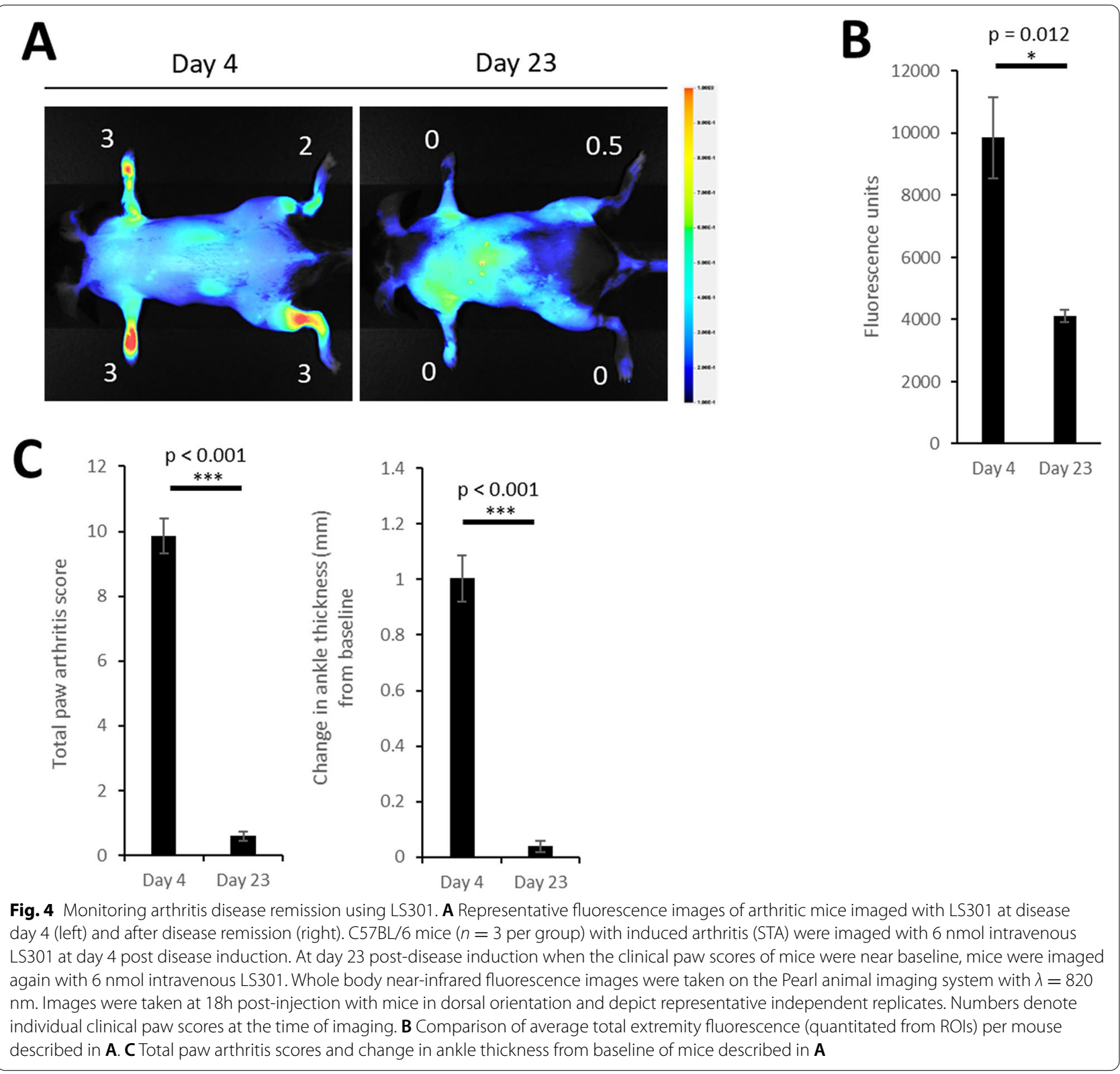

\section{LS301 fluorescence correlates with arthritis disease severity in affected limbs}

We next assessed the utility of LS301 as an imaging modality for monitoring disease activity. In a cohort of mice with STA, total fluorescence in each affected limb area encompassing the animal's upper extremities (all structures including and distal to the wrist) or lower extremities (all structures including and distal to the ankle), as determined using the Pearl software via ROI quantification (Fig. S3), was plotted against clinical paw scores and ankle thickness measurements. As described in Methods, experiments were performed in blinded fashion where the technician responsible for clinical assessment of paw score and ankle measurements was blinded to treatment groups. We found a significant positive correlation between LS301 fluorescence and disease severity by both parameters $(r=0.86$ and $r=0.80$ respectively) (Fig. 3). In addition, LS301 fluorescence signal successfully discriminated between diseased and healthy (non-diseased) extremities using a threshold total clinical paw score of 1 . These results demonstrate the potential of LS301 as a useful tool for monitoring the severity of disease activity and progression. 


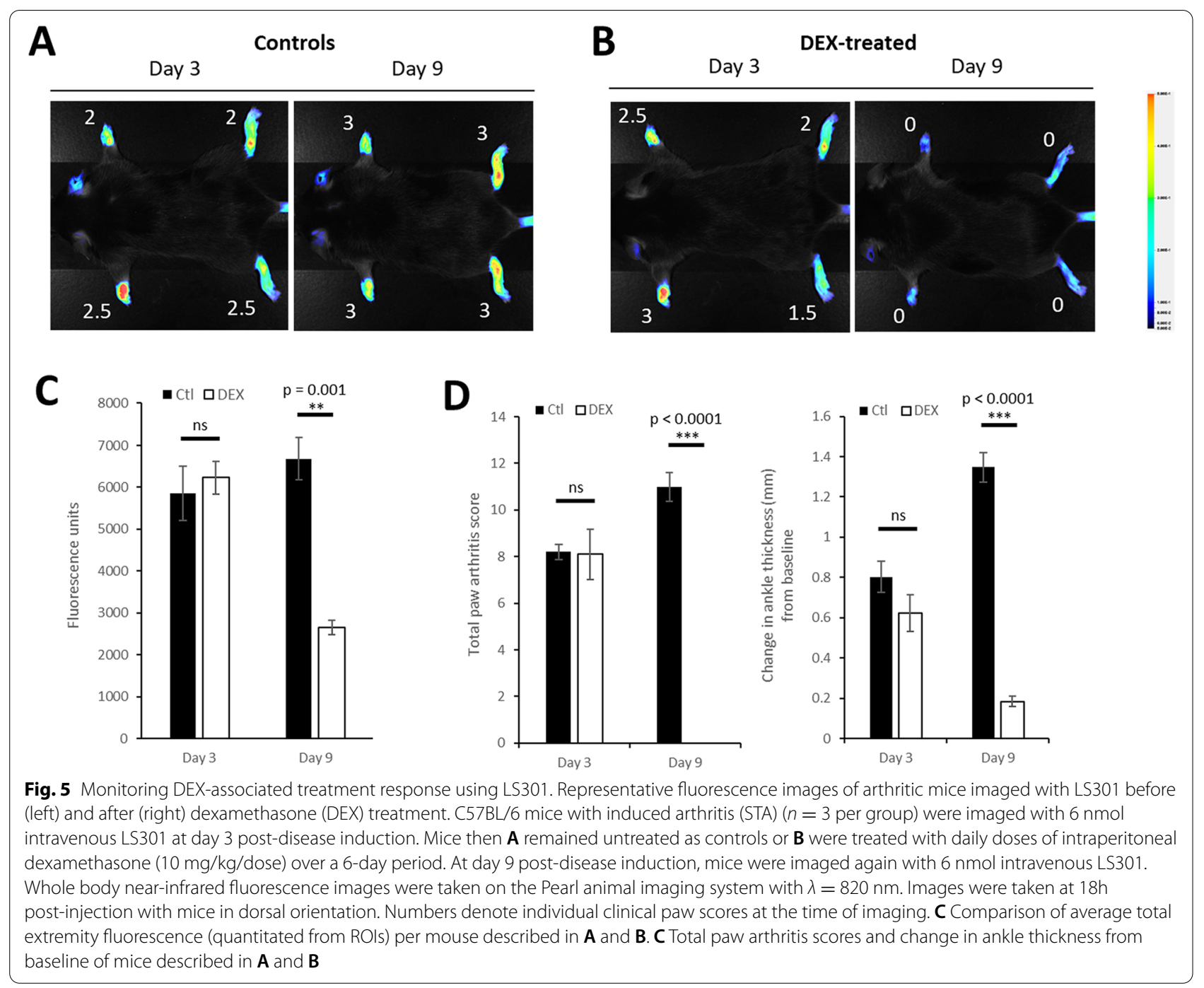

\section{LS301 tracks disease progression and regression}

Current paradigms in RA management generally require trial periods on a time scale of months before the therapeutic response of a patient to DMARDs can be determined via clinical scoring, imaging, and/or inflammatory markers. This delay leads to increased risks of disease progression during this interval as well as unnecessary drug toxicities and financial expenses. Therefore, an imaging technique capable of reporting early response to drug treatment would have a significant translational impact. As a prelude to further studies, we confirmed that LS301 administration alone at the imaging dose $(6$ nmol) did not significantly affect disease progression (Fig. S4). First, to assess the use of LS301 in monitoring disease activity, we imaged mice with STA near the peak of their disease (day 4 post serum transfer) using LS301. At day 23, when clinical scores had returned to their baseline (pre-disease induction) levels, the mice were imaged again using LS301. As shown in Fig. 4, LS301 fluorescence correlated with disease progression and regression in these mice as orthogonally confirmed by clinical paw scoring.

Next, to evaluate the use of LS301 in monitoring early treatment response, we imaged mice with STA (day 3 post disease induction) using LS301. Mice then received either no treatment (control) or six daily treatments of dexamethasone (DEX). After the conclusion of the treatment cycle (day 9 post disease induction), mice were imaged again with LS301. While control mice continued to show disease progression as assessed by a high level of LS301 fluorescence and clinical paw scores (Fig. 5A, C-D), mice treated with DEX showed reductions in disease severity that correlated closely with LS301 fluorescence (Fig. 5B, $\mathrm{C}-\mathrm{D})$. Taken together, these results demonstrate the utility of LS301 fluorescence for monitoring early treatment response for RA. 

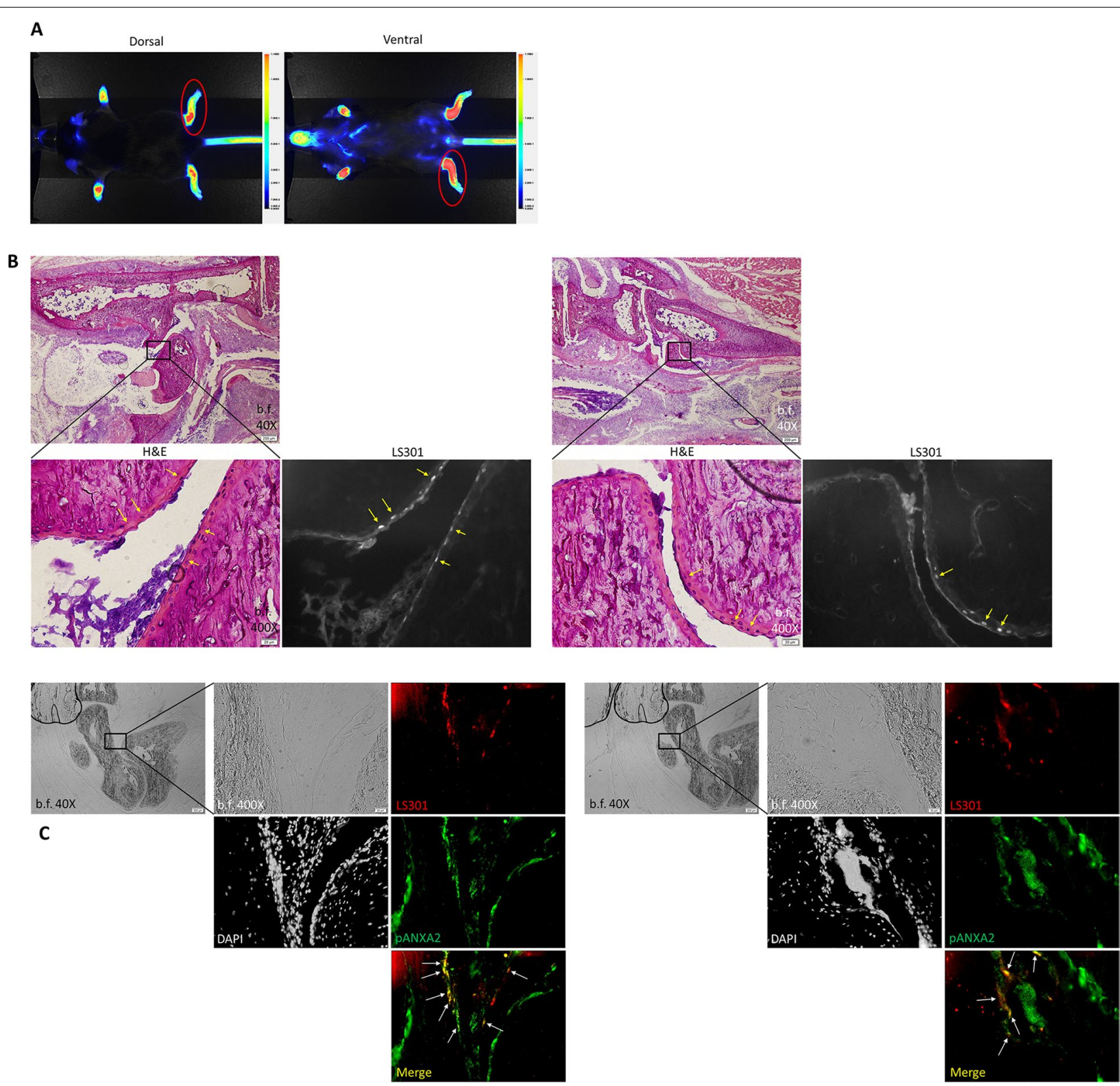

Fig. 6 Cellular localization of LS301 in the arthritic mouse paws. C57BL/6 mice with serum transfer arthritis were intravenously injected with 6 nmol LS301 at day 4 post disease induction. $6 \mathrm{~h}$ after LS301 injection, whole body near-infrared fluorescence images were takePlease check if the figure captions are presented correctly.n on the Pearl animal imaging system with $\lambda=820 \mathrm{~nm}$, and subsequently paws and ankles were harvested and frozen for sectioning. Sections were stained with H\&E, or left unstained and examined for LS301 fluorescence by microscopy under the cypate channel (Ex/Em 775 $\pm 25 \mathrm{~nm} / 845 \pm 28 \mathrm{~nm}$ ) (red) and/or viewed for pANXA2 (AlexaFluor 594 fluorescence) (green) under the Texas Red channel (Ex/Em 562 $20 \mathrm{~nm} / 624 \pm 20 \mathrm{~nm}$ ). Images are representative of results from at least two independent experiments. A Example Pearl near-infrared fluorescence images of mice immediately prior to limb harvest. Red circles denote representative examples of the limb area harvested for sectioning. B H\&E and fluorescence images from corresponding regions of articular cartilage in mouse ankle. Arrows indicate example regions of LS301 accumulation. C Fluorescence microscopy images from corresponding regions of articular cartilage in mouse ankle. Sections were stained with DAPI and anti-pANXA2 Ab/AlexaFluor 594-conjugated secondary Ab. Shown are DAPI (grayscale), LS301 fluorescence (red), and pANXA2 (green). Arrows denote LS301-pANXA2 colocalization

\section{LS301 accumulates within chondrocytes and articular cartilage in arthritic mice}

To assess the tissue distribution of LS301, we administered LS301 to mice with STA and harvested mouse ankle tissues for fluorescent and immunohistochemical (IHC) analyses (Fig. 6A). H\&E-stained sections of ankles showed clear localization of LS301 fluorescence within chondrocytes and articular cartilage by superimposition 


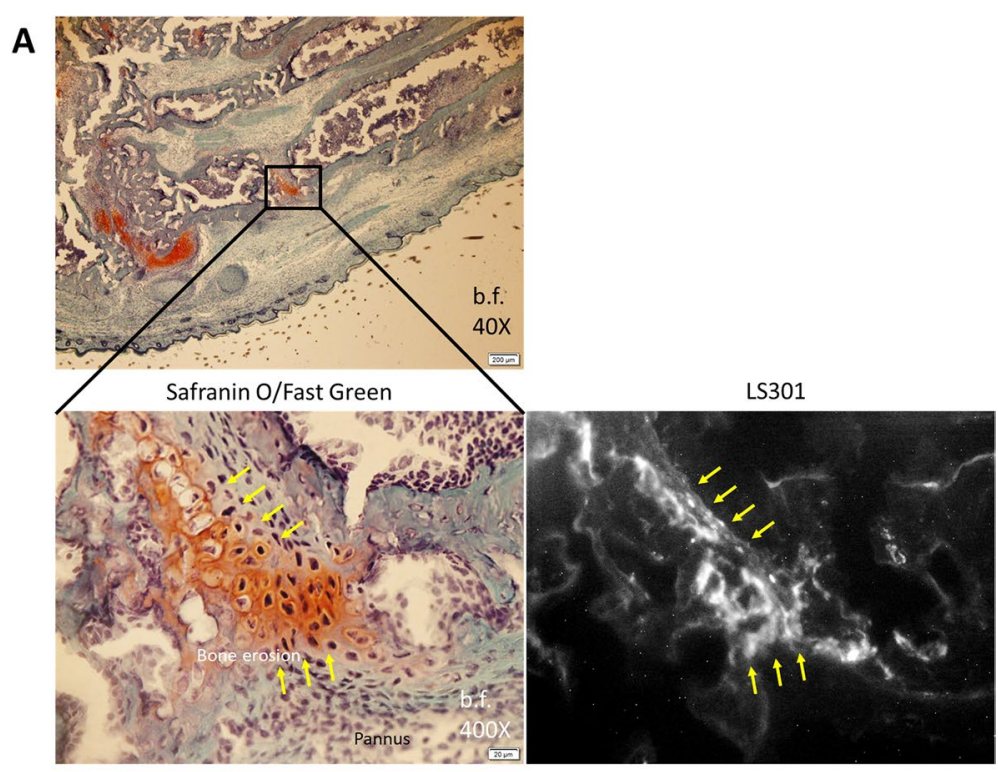

B

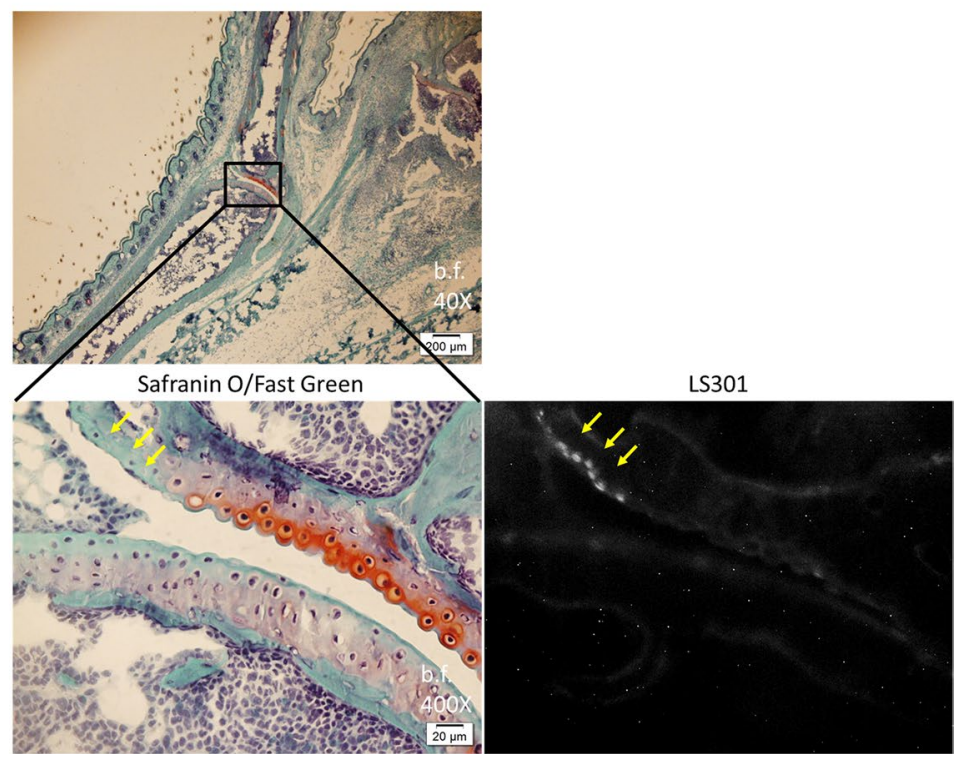

Fig. 7 LS301 association with cartilage damage. K/BxN F1 mice (6-7 weeks old) ( $n=2$ mice per group) were injected intravenously with 6 nmol LS301. Six hours after LS301 injection, paws and ankles were harvested and frozen for sectioning. Sections were stained with Safranin O/Fast Green and viewed for LS301 fluorescence by microscopy under the cypate channel (Ex/Em 775 $\pm 25 \mathrm{~nm} / 845 \pm 28 \mathrm{~nm})$. A Images from regions associated with pannus/bone erosion. Arrows indicate areas of LS301 accumulation. B Images showing preferential association of LS301 with regions of cartilage damage (loss of Safranin O staining). Arrows indicate areas of LS301 accumulation

of fluorescence images with H\&E-stained images (Fig. 6B). In contrast, minimal or no LS301 signal above background was detected in other tissue regions including skin, connective tissue, muscle, bone, or bone marrow (Figs. S5 and S6). Previously, we found that LS301 binds with high affinity to pANXA2, which is known to be upregulated in arthritic cartilage [36, 37, 43]. IHC staining of ankle sections for pANXA2 revealed significant colocalization of LS301 fluorescence with pANXA2 expression within articular cartilage (Fig. 6C).

To further evaluate whether there is a correlation between cartilage damage and LS301 accumulation in the joint, we examined paw sections stained with Safranin O. We found that LS301 localizes to areas of pannus/bone erosion (Fig. 7A). Moreover, LS301 preferentially localizes to regions of damaged cartilage, as evidenced by loss of Safranin O 
staining (Fig. 7B). These results indicate that LS301 targets chondrocytes in areas of damaged cartilage.

\section{Discussion}

In lieu of the current trial-and-error practice for choosing RA therapeutics, a system for rapid assessment of arthritis treatment efficacy would enable proper drug selection at the crucial early stages when the disease course is most amenable to drug treatment. Such a system would also provide a much-needed platform for rapid screening and development of novel therapeutics. We describe here a novel near-infrared molecular imaging agent termed LS301, which was previously shown to target pANXA 2 [31], and demonstrate its utility in monitoring RA disease activity, progression, and early (6 days) molecular response to treatment in the context of fluorescence imaging. We have shown in mouse models of RA that the therapeutic response to drug treatment can be tracked on a time span of several days using LS301, which if successfully translated to clinical application would represent a substantial improvement over the months required by the current scoring and imaging methods. Near-infrared FI does not involve ionizing radiation, and thus benefits from increased safety relative to imaging methods such as PET, conventional radiography, and CT. A further translational advantage of LS301 compared to other targeted fluorescent agents is that this agent has advanced beyond preclinical testing and is currently undergoing a clinical trial for cancer imaging (\# NCT02807597) at the time of this report.

Of note, we observed that LS301 localizes preferentially in chondrocytes within the arthritic articular cartilage (Fig. 6b). As the sole producers of cartilaginous matrix and as mediators of inflammation, chondrocytes represent an important future cellular target for therapy in RA as well as osteoarthritis [44-46]. Chondrocytes have been a relatively overlooked target in fluorescence imaging approaches for RA, which have typically focused on inflammatory cells such as macrophages [27, 28]. Thus far, efforts to deliver drugs to chondrocytes have had limited success due to rapid clearance of molecules from the joint space following injections, the avascular nature of cartilage tissue, and the location of chondrocytes in the relatively inaccessible middle and deep zones of cartilage $[47,48]$. The observed penetration of LS301 into chondrocytes raises the possibility of specific drug delivery to these cells via conjugation with LS301. Further studies are warranted to elucidate the unique mechanism(s) enabling LS301 to traverse the biological barriers posed by the cartilaginous tissues.

In our biodistribution studies, we note that there exists some degree of natural inter-individual variability in organ LS301 uptake. For example, in a proportion of the mice, a possible nonsignificant trend toward increased kidney signal in arthritic mice when compared to controls was observed. It was previously reported that G6PIantibody immune complexes, which are a key component in the pathogenesis of our serum transfer arthritis model, also localize to the kidney glomeruli [49]. It is possible that in some individuals, such a process could result in inflammation/elevated pANXA2 expression and some degree of LS301 accumulation in this region.

In line with a shift toward precision medicine, strategies for targeting drugs to sites of inflammation could enhance the potential of existing rheumatologic drugs by increasing local delivery and reducing off-target toxicity. Although intra-articular injection of therapeutics can achieve high local concentrations, this approach becomes impractical in cases where multiple joints are involved such as in RA. Our observation that LS301 accumulates in target areas of inflammation following administration suggests its potential to circumvent these challenges. The unique chemical structure of LS301 (Fig. S1) enables it to readily serve as a covalent drug carrier via linkage with small molecule drugs or peptides. Further development of LS301 and its conjugates may enable the development of novel first-in-class theranostic agents not only for RA but also for $\mathrm{OA}$ and other arthritides.

\section{Conclusions}

We demonstrate that FI using the pANXA2-targeting agent LS301 can be used to monitor the progression, remission, and early response to drug treatment in mouse models of RA. The observed selectivity of LS301 for arthritic lesions and the association of LS301 with chondrocytes in vivo provides a novel potential avenue for molecularly targeted imaging and drug evaluation.

\section{Abbreviations}

RA: Rheumatoid arthritis; DMARDs: Disease-modifying antirheumatic drugs; $\mathrm{Fl}$ : Fluorescence imaging; ICG: Indocyanine green; pANXA2: Tyr23-phosphorylated annexin A2; STA: Serum transfer arthritis.

\section{Supplementary Information}

The online version contains supplementary material available at https://doi. org/10.1186/s13075-021-02643-3.

Additional file 1: Figure S1. Chemical structure of LS301.

Additional file 2: Figure S2. LS301 time course imaging in arthritic mice. Mice with spontaneous K/BxN arthritis (9-10 weeks old) ( $n=1$ per group) were injected intravenously with $6 \mathrm{nmol}$ LS301. Whole body near-infrared fluorescence images were taken at the indicated times on the Pearl animal imaging system with $\lambda=820 \mathrm{~nm}$.

Additional file 3: Figure S3. Regions of interest (ROls) used for quantitation of fluorescence in individual arthritic limbs. Representative example of regions of interest (ROI) encompassing mouse upper extremities (all structures distal to and including the wrist) and lower extremities (all 
structures distal to and including the ankle) that were quantitated for LS301 fluorescence using the Pearl animal imaging system software.

Additional file 4: Figure S4. Effect of imaging-dose ( $6 \mathrm{nmol}$ ) LS301 on arthritic disease progression. C57BL/6 mice with serum transfer arthritis ( $n=5$ per group) were treated daily with $6 \mathrm{nmol}$ intravenous LS301 from days 0 through 4 post disease induction, with daily 4 h post-injection imaging. Whole body near-infrared fluorescence images were taken on Pearl Imaging System with $\lambda=820 \mathrm{~nm}$. Aggregate clinical paw scores were determined daily. Arrows denote the timing of LS301 treatments.

Additional file 5: Figure S5. Controls for immunohistochemical staining C57BL/6 mice $(n=1)$ with serum induced arthritis were injected intravenously with $6 \mathrm{nmol}$ LS301 at day 4 post disease induction. $6 \mathrm{~h}$ after LS301 injection, whole body near-infrared fluorescence images were taken on the Pearl animal imaging system with $\lambda=820 \mathrm{~nm}$, and subsequently paws and ankles were harvested and frozen for sectioning. Sections were incubated with AlexaFluor 594-conjugated secondary antibody only and viewed for fluorescence by microscopy under the Texas Red channel (EX/ Em 562 $\pm 20 \mathrm{~nm} / 624 \pm 20 \mathrm{~nm}$ ).

Additional file 6: Figure S6. Tissue distribution of LS301 in extraarticular regions of the mouse extremity. C57BL/6 mice with serum transfer arthritis were injected intravenously with $6 \mathrm{nmol}$ LS301 at day 4 post disease induction. $6 \mathrm{~h}$ after LS301 injection, paws and ankles were harvested and frozen for sectioning. Sections were stained with H\&E and viewed for LS301 fluorescence by microscopy under the cypate channel (Ex/Em $775 \pm 25 \mathrm{~nm} / 845 \pm 28 \mathrm{~nm}$ ) (red). Shown are representative H\&E and fluorescence images from the indicated tissues in mouse ankle. Images are representative of at least two independent experiments. (A) Skin/dermis. (B) Connective tissue. (C) Muscle. (D) Bone. (E) Bone marrow.

Additional file 7: Figure S7. Relative expression levels of pANXA2 and ANXA2 in ankle and foot tissues of arthritic mice. C57BL/6 mice with serum transfer arthritis ( $n=1$ per group) were sacrificed at day 8 post disease induction and ankle and foot tissue were harvested, homogenized and subjected to immunoblotting analysis for PANXA2 and ANXA2. Left: Immunoblot data for pANXA2 and ANXA2 in arthritic vs. control mouse tissue. Right: Corresponding signal quantitation using ImageJ software.

Additional file 8: Figure S8. Biodistribution of LS301 shown on different scales. Shown is an example LS301 organ biodistribution from C57BL/6 mice with STA or control mice injected intravenously with LS301 ( $n=4$ per group), shown at two different scales. Fluorescence images shown were acquired using the Pearl Small Animal Imager. Left: Arthritic mouse LS301 organ biodistribution; right: control mouse LS301 organ biodistribution.

Additional file 9: Figure S9. Representative image depicting organ $\mathrm{RO}$ delineation on the Pearl Small Animal Imager software. Shown is an example LS301 organ biodistribution from C57BL/6 mice with STA injected intravenously with LS301 $(n=4)$. ROls were drawn using the freehand shape tool on the Pearl Small Animal Imager.

Additional file 10: Figure S10. Raw data from ROI quantitation (Fig. 2B).

\section{Acknowledgements}

We would like to thank Louise Berryman for providing administrative support, Baogang Xu for assistance with immunoblot studies, and Crystal Idleburg of the Musculoskeletal Histology and Morphometry Core (Washington University School of Medicine) for providing tissue sectioning and staining services.

\section{Authors' contributions}

SDT, CTNP, and SA conceived and designed all studies; SDT, LES, GS, AK, and CTNP performed the imaging, biological, and histological studies; KSG, RT, and $\mathrm{KL}$ performed the chemical syntheses and purifications. The authors read and approved the final manuscript.

\section{Funding}

This study was supported by grants from the National Institutes of Health (AR076820, AR073752, R01 EB030987, R01 CA260855, U54 CA199092, R01 EB021048, P30 CA091842, P30 CA091842-19S3, P30 AR073752, R01 AR067491, S10 OD027042, S10 OD016237, S10 RR031625, and S10 OD020129), the Department of Defense Breast Cancer Research Program
(W81XWH-16-1-0286), the Siteman Investment Program (SIP) Research Development Award, and VA Merit Review 101 BX005075 (CTNP).

\section{Availability of data and materials}

The datasets used and/or analyzed during the current study are available from the corresponding author on reasonable request.

\section{Declarations}

\section{Ethics approval and consent to participate}

All animal experiments were performed in compliance with guidelines and protocols approved by the Division of Comparative Medicine at Washington University in St. Louis. The animal protocol is subjected to annual review and approval by The Animal Studies Committee of Washington University.

\section{Consent for publication}

Not applicable.

\section{Competing interests}

The authors declare that they have no competing interests, except that SA is a co-inventor on a US patent issued to Washington University covering LS301, which has been licensed for cancer imaging. The patent may become the subject of a licensing agreement in the future.

\section{Author details}

'Departments of Radiology, Washington University School of Medicine, St Louis, MO 63110, USA. ${ }^{2}$ Division of Rheumatology, Washington University School of Medicine, St Louis, MO 63110, USA. ${ }^{3}$ Departments of Biochemistry and Molecular Biophysics, Washington University School of Medicine, St Louis, MO 63110, USA. ${ }^{4}$ Departments of Biomedical Engineering, Washington University School of Medicine, St Louis, MO 63110, USA.

Received: 2 June 2021 Accepted: 4 October 2021

Published online: 25 October 2021

\section{References}

1. Hunter TM, Boytsov NN, Zhang X, Schroeder K, Michaud K, Araujo AB. Prevalence of rheumatoid arthritis in the United States adult population in healthcare claims databases, 2004-2014. Rheumatol Int. 2017;37(9):1551-7.

2. Almutairi K, Nossent J, Preen D, Keen H, Inderjeeth C. The global prevalence of rheumatoid arthritis: a meta-analysis based on a systematic review. Rheumatol Int. 2020.

3. Deshpande BR, Katz JN, Solomon DH, Yelin EH, Hunter DJ, Messier SP, et al. Number of Persons with symptomatic knee osteoarthritis in the US: impact of race and ethnicity, age, sex, and obesity. Arthritis Care Res (Hoboken). 2016;68(12):1743-50.

4. Vina ER, Kwoh CK. Epidemiology of osteoarthritis: literature update. Curr Opin Rheumatol. 2018;30(2):160-7.

5. Lipsky PE, van der Heijde DM, St Clair EW, Furst DE, Breedveld FC, Kalden $J R$, et al. Infliximab and methotrexate in the treatment of rheumatoid arthritis. Anti-Tumor Necrosis Factor Trial in Rheumatoid Arthritis with Concomitant Therapy Study Group. N Engl J Med. 2000;343(22):1594-602.

6. Smolen JS, Avila JC, Aletaha D. Tocilizumab inhibits progression of joint damage in rheumatoid arthritis irrespective of its anti-inflammatory effects: disassociation of the link between inflammation and destruction. Ann Rheum Dis. 2012;71(5):687-93.

7. Smolen JS, Landewe R, Bijlsma J, Burmester G, Chatzidionysiou K, Dougados $M$, et al. EULAR recommendations for the management of rheumatoid arthritis with synthetic and biological disease-modifying antirheumatic drugs: 2016 update. Ann Rheum Dis. 2017;76(6):960-77.

8. Salomon-Escoto K, Kay J. The "Treat to Target" Approach to Rheumatoid Arthritis. Rheum Dis Clin North Am. 2019;45(4):487-504.

9. Romao VC, Fonseca JE. Major Challenges in Rheumatology: Will We Ever Treat Smarter, Instead of Just Harder? Front Med (Lausanne). 2019;6:144.

10. Guidelines for the management of rheumatoid arthritis. American College of Rheumatology Ad Hoc Committee on Clinical Guidelines. Arthritis Rheum. 1996;39(5):713-22. 
11. Wijbrandts CA, Tak PP. Prediction of Response to Targeted Treatment in Rheumatoid Arthritis. Mayo Clin Proc. 2017;92(7):1129-43.

12. Grigor C, Capell H, Stirling A, McMahon AD, Lock P, Vallance R, et al. Effect of a treatment strategy of tight control for rheumatoid arthritis (the TICORA study): a single-blind randomised controlled trial. Lancet. 2004;364(9430):263-9.

13. Wakefield RJ, Gibbon WW, Conaghan PG, O'Connor P, McGonagle D, Pease $C$, et al. The value of sonography in the detection of bone erosions in patients with rheumatoid arthritis: a comparison with conventional radiography. Arthritis Rheum. 2000;43(12):2762-70.

14. Viitanen R, Moisio O, Lankinen P, Li XG, Koivumaki M, Suilamo S, et al. First-in-Humans Study of (68)Ga-DOTA-Siglec-9, a PET Ligand Targeting Vascular Adhesion Protein 1. J Nucl Med. 2021;62(4):577-83.

15. Monti S, Montecucco C, Bugatti S, Caporali R. Rheumatoid arthritis treatment: the earlier the better to prevent joint damage. RMD Open. 2015;1(Suppl 1):e000057.

16. Fischer T, Ebert B, Voigt J, Macdonald R, Schneider U, Thomas A, et al. Detection of rheumatoid arthritis using non-specific contrast enhanced fluorescence imaging. Acad Radiol. 2010;17(3):375-81.

17. Fischer T, Gemeinhardt I, Wagner S, Stieglitz DV, Schnorr J, Hermann KG, et al. Assessment of unspecific near-infrared dyes in laser-induced fluorescence imaging of experimental arthritis. Acad Radiol. 2006;13(1):4-13.

18. Glimm AM, Sprenger LI, Haugen IK, Mansmann U, Hermann S, HaupI T, et al. Fluorescence optical imaging for treatment monitoring in patients with early and active rheumatoid arthritis in a 1-year follow-up period. Arthritis Res Ther. 2019;21(1):209.

19. Kisten Y, Gyori N, Af Klint E, Rezaei H, Levitsky A, Karlsson A, et al. Detection of clinically manifest and silent synovitis in the hands and wrists by fluorescence optical imaging. RMD Open. 2015;1(1):e000106.

20. Krohn M, Ohrndorf S, Werner SG, Schicke B, Burmester GR, Hamm B, et al. Near-infrared Fluorescence Optical Imaging in Early Rheumatoid Arthritis: A Comparison to Magnetic Resonance Imaging and Ultrasonography. J Rheumatol. 2015;42(7):1112-8.

21. Meier R, Krug C, Golovko D, Boddington S, Piontek G, Rudelius M, et al. Indocyanine green-enhanced imaging of antigen-induced arthritis with an integrated optical imaging/radiography system. Arthritis Rheum 2010;62(8):2322-7.

22. Schafer VS, Hartung W, Hoffstetter P, Berger J, Stroszczynski C, Muller $M$, et al. Quantitative assessment of synovitis in patients with rheumatoid arthritis using fluorescence optical imaging. Arthritis Res Ther. 2013;15(5):R124.

23. Werner SG, Langer HE, Ohrndorf S, Bahner M, Schott P, Schwenke C, et al. Inflammation assessment in patients with arthritis using a novel in vivo fluorescence optical imaging technology. Ann Rheum Dis. 2012;71(4):504-10.

24. Werner SG, Langer HE, Schott P, Bahner M, Schwenke C, Lind-Albrecht $\mathrm{G}$, et al. Indocyanine green-enhanced fluorescence optical imaging in patients with early and very early arthritis: a comparative study with magnetic resonance imaging. Arthritis Rheum. 2013;65(12):3036-44.

25. Jamar F, Houssiau FA, Devogelaer JP, Chapman PT, Haskard DO, Beaujean $V$, et al. Scintigraphy using a technetium 99m-labelled anti-Eselectin Fab fragment in rheumatoid arthritis. Rheumatology (Oxford). 2002:41(1):53-61.

26. Bhatnagar S, Khera E, Liao J, Eniola V, Hu Y, Smith DE, et al. Oral and Subcutaneous Administration of a Near-Infrared Fluorescent Molecular Imaging Agent Detects Inflammation in a Mouse Model of Rheumatoid Arthritis. Sci Rep. 2019;9(1):4661.

27. Chen WT, Mahmood U, Weissleder R, Tung CH. Arthritis imaging using a near-infrared fluorescence folate-targeted probe. Arthritis Res Ther. 2005;7(2):R310-7.

28. Hansch A, Frey O, Sauner D, Hilger I, Haas M, Malich A, et al. In vivo imaging of experimental arthritis with near-infrared fluorescence. Arthritis Rheum. 2004;50(3):961-7.

29. Cho SS, Salinas R, Lee JYK. Indocyanine-Green for Fluorescence-Guided Surgery of Brain Tumors: Evidence, Techniques, and Practical Experience. Front Surg. 2019;6:11.
30. Wang H, Li X, Tse BW, Yang H, Thorling CA, Liu Y, et al. Indocyanine green-incorporating nanoparticles for cancer theranostics. Theranostics. 2018;8(5):1227-42.

31. Shen D, Xu B, Liang K, Tang R, Sudlow GP, Egbulefu C, et al. Selective imaging of solid tumours via the calcium-dependent high-affinity binding of a cyclic octapeptide to phosphorylated Annexin A2. Nat Biomed Eng. 2020;4(3):298-313.

32. Bharadwaj $A$, Bydoun $M$, Holloway $R$, Waisman D. Annexin $A 2$ heterotetramer: structure and function. Int J Mol Sci. 2013;14(3):6259-305.

33. Song C, Zhou X, Dong Q, Fan R, Wu G, Ji B, et al. Regulation of inflammatory response in human chondrocytes by lentiviral mediated RNA interference against S100A10. Inflamm Res. 2012;61(11):1219-27.

34. Deora AB, Kreitzer G, Jacovina AT, Hajjar KA. An annexin 2 phosphorylation switch mediates p11-dependent translocation of annexin 2 to the cell surface. J Biol Chem. 2004;279(42):43411-8.

35. Zheng L, Foley K, Huang L, Leubner A, Mo G, Olino K, et al. Tyrosine 23 phosphorylation-dependent cell-surface localization of annexin A2 is required for invasion and metastases of pancreatic cancer. PLoS One. 2011;6(4):e19390

36. Yi J, Zhu Y, Jia Y, Jiang $H$, Zheng $X$, Liu D, et al. The Annexin a2 Promotes Development in Arthritis through Neovascularization by Amplification Hedgehog Pathway. PLoS One. 2016;11(3):e0150363.

37. Zhao W, Zhang C, Shi M, Zhang J, Li M, Xue X, et al. The discoidin domain receptor 2/annexin A2/matrix metalloproteinase 13 loop promotes joint destruction in arthritis through promoting migration and invasion of fibroblast-like synoviocytes. Arthritis Rheumatol. 2014;66(9):2355-67.

38. Monach $\mathrm{P}$, Hattori $\mathrm{K}$, Huang $\mathrm{H}$, Hyatt E, Morse J, Nguyen L, et al. The K/BxN mouse model of inflammatory arthritis: theory and practice. Methods Mol Med. 2007;136:269-82.

39. Monach PA, Mathis D, Benoist C: The K/BxN arthritis model. Curr Protoc Immunol 2008, Chapter 15:Unit 1522

40. Zhou HF, Chan HW, Wickline SA, Lanza GM, Pham CT. Alphavbeta3targeted nanotherapy suppresses inflammatory arthritis in mice. FASEB J. 2009;23(9):2978-85.

41. Zhou HF, Hu G, Wickline SA, Lanza GM, Pham CT. Synergistic effect of antiangiogenic nanotherapy combined with methotrexate in the treatment of experimental inflammatory arthritis. Nanomedicine (Lond). 2010;5(7):1065-74

42. Rosner B. Fundamentals of Biostatistics. 7th ed. Boston: Brooks/Cole; 2011

43. Achilefu S, Dorshow RB, Bugaj JE, Rajagopalan R. Novel receptor-targeted fluorescent contrast agents for in vivo tumor imaging. Invest Radiol. 2000:35(8):479-85.

44. Tseng CC, Chen YJ, Chang WA, Tsai WC, Ou TT, Wu CC, et al. Dual Role of Chondrocytes in Rheumatoid Arthritis: The Chicken and the Egg. Int J Mol Sci. 2020;21:3

45. Otero M, Goldring MB. Cells of the synovium in rheumatoid arthritis. Chondrocytes. Arthritis Res Ther. 2007;9(5):220.

46. Evans CH. Catering to chondrocytes. Sci Transl Med. 2018;10:469.

47. Geiger BC, Wang S, Padera RF Jr, Grodzinsky AJ, Hammond PT. Cartilagepenetrating nanocarriers improve delivery and efficacy of growth factor treatment of osteoarthritis. Sci Transl Med. 2018;10:469.

48. Bajpayee AG, Quadir MA, Hammond PT, Grodzinsky AJ. Charge based intra-cartilage delivery of single dose dexamethasone using Avidin nanocarriers suppresses cytokine-induced catabolism long term. Osteoarthritis Cartilage. 2016;24(1):71-81.

49. Kamradt T, Schubert D. The role and clinical implications of G6PI in experimental models of rheumatoid arthritis. Arthritis Res Ther. 2005;7(1):20-8.

\section{Publisher's Note}

Springer Nature remains neutral with regard to jurisdictional claims in published maps and institutional affiliations. 